\title{
XIII International Conference on Hole Burning, Single Molecule, and Related Spectroscopies: Science and Applications (HBSM-2018): preface
}

\author{
Andrei Naumov*, Maxim Gladush, and Kamil Karimullin \\ ${ }^{1}$ Institute for Spectroscopy RAS, 108840 Moscow, Troitsk, Russia \\ ${ }^{2}$ Moscow State Pedagogical University, 119991 Moscow, Russia
}

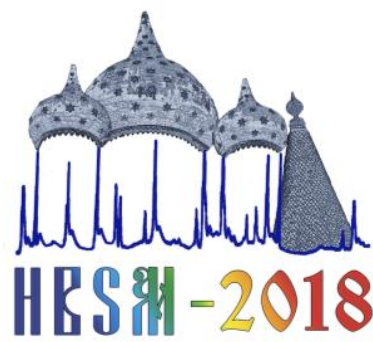

\section{International Conference on HOLE BURNING, SINGLE MOLECULE AND RELATED SPECTROSCOPIES: SCIENCE AND APPLICATIONS}

August 6-12, 2018 Suzdal - Moscow, Russia
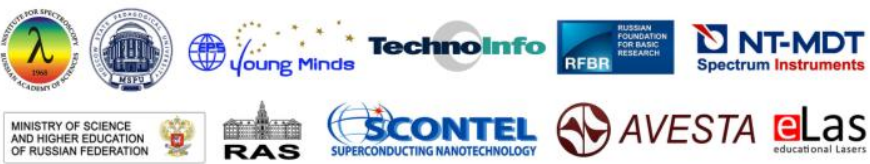

\section{Foreword}

XIII International Conference on Hole Burning, Single Molecule, and Related Spectroscopies: Science and Applications (HBSM-2018) was organized by the Institute for Spectroscopy of the Russian Academy of Sciences (ISAN; Troitsk, Moscow) with the Moscow State Pedagogical University (MSPU) in Suzdal from $6^{\text {th }}$ till $10^{\text {th }}$ August 2018, and in Moscow and Troitsk on $11^{\text {th }}$ August 2018.

Laser selective spectroscopy has a particular significance and value for the Russian scientific community. The emergence of quasi-linear spectra of complex organic compounds in specially selected solvents at low cryogenic temperatures known as "Shpol'skii matrices" was first observed in 1952 by the members of Prof. Eduard Shpol'skii's group from the Department of Theoretical Physics of the Moscow State Pedagogical Institute. In 1972, a former student of Eduard Shpol'skii, Prof. Roman Personov, and his colleagues at the Institute for Spectroscopy of the USSR Academy of Sciences demonstrated successful site-selective laser excitation of narrow luminescence spectra in organic molecules. Two years later, in 1974, Personov's group with their colleagues from the Institute of Physics in Tartu, Estonia (USSR) conducted the fundamental experiments on the persistent spectral hole burning. That work formed the basis of the laser site-selective spectroscopy of molecules which later evolved in singlemolecule spectroscopy.

HBSM was known under a few different names. It started in 1987 in Tallin, Estonia (USSR) as "All-Union Symposium on Modern Methods of Laser Spectroscopy of Molecules in Low-Temperature Media". The second meeting took place in Monterey (California, USA, 1991) as "Persistent Spectral Hole Burning: Science and Applications" and the third meeting became "Spectral Hole Burning and Luminescence Line Narrowing:

\footnotetext{
* Corresponding author: naumov@isan.troitsk.ru; web-page: www.single-molecule.ru
} 
Science and Applications" and moved to Ascona (Switzerland, 1992). Later it was organized as "International conferences on Hole-Burning and Related Spectroscopies (HBRS): Science and Applications" in Tokyo (Japan, 1994), Brainerd (Minnesota, USA, 1996) and Hourtin (France, 1999). In 2001, the conference finally found its current name "Hole Burning, Single Molecule, and Related Spectroscopies (HBSM): Science and Applications". It paved its path through Taipei (Taiwan, 2001), Bozeman (Montana, USA, 2003), Aussois (France, 2006), Palm Cove (Australia, 2009), Tübingen (Germany, 2012) and Tartu (Estonia, 2015). This conference series has been exceptionally successful in bringing together participants from the leading research teams around the world to discuss the latest developments in the fundamental science and the applications of site-selective and single-molecule spectroscopies and numerous related topics (condensed matter physics and physical chemistry, nanoscopy, quantum dots, rear-earth ions, nanodiamonds, biophysics, medical nanodiagnostics, quantum technologies, photovoltaics, on-chip technologies).

The scientific program of the conference included 3 plenary lectures, 19 invited talks, 34 oral talks, 41 poster talks, authored in total by 189 scientists from 21 countries (Australia, Belarus, Belgium, Canada, China, Denmark, Estonia, France, Germany, India, Israel, Italy, Japan, Netherlands, Poland, Russia, Serbia, Sweden, Switzerland, Ukraine, USA).

The students and young scientists at HBSM-2018 displayed their research findings in two poster sections while some were selected to give oral presentations. Their participation included a competition for the Best Student/Young Scientist Talk Award. The winners of the awards were Andrey Anisimov (Ioffe Institute RAS, St. Petersburg, Russia) for poster talk and Alexey Tiranov (University of Geneva, Switzerland) for oral talk. As an additional side-event for the young scientists there was a meeting organized by the "Moscow Pedagogical State University Young Minds Section" of the European Physical Society.

The HBSM-2018 was accompanied with the exhibition organized by the sponsoring companies, where the modern equipment for optics, spectroscopy and related areas from the world-wide leading manufacturers was demonstrated. The organizers acknowledge financial support from the Russian Foundation for Basic Research (project No 18-0220081; www.rfbr.ru) and hi-tech companies working in the field of development, manufacturing and marketing of modern scientific equipment: Educational Lasers - eLas (laser and photonics teaching systems; Freiburg, Germany; www.e-las.com); Technoinfo Ltd (analytical, laboratory and technological equipment; Moscow, Russia; www.technoinfo.co.uk) which is official representative of PicoQuant (pulsed diode lasers, time-resolved data acquisition, single photon counting, and fluorescence instrumentation; Berlin, Germany; www.picoquant.com) in Russia; Superconducting nanotechnology (single photon detector systems for VIS and NIR spectral range; Moscow, Russia; www.scontel.ru); NT-MDT Spectrum Instruments (atomic force microscopes and its combinations with ultrahigh resolution spectroscopy for nanotechnology and applications; Moscow, Russia; www.ntmdt-si.com); Avesta Ltd (lasers and optical components; Moscow, Russia; www.avesta.ru).

This issue of the EPJ Web of Conferences has collected the brief information about HBSM-2018 as well as the summaries of plenary lectures, conference talks and poster presentations. The issue is recommended for specialists who work in the field optics and spectroscopy, as well as for M.S. and Ph.D. students. The full program and details about the conference can be found on the web-site www.hbsm2018.ru). The next XIV International Conference on Hole Burning, Single Molecule, and Related Spectroscopies: Science and Applications (HBSM-2021) will be in Banz Abbey (Bad Staffelstein, Bavaria) in Germany.

\section{International Advisory and Program Committee}

Thomas Basché

Christian von Borczyskowski

Marc Brecht
Johannes Gutenberg University Mainz, Germany

Chemnitz University of Technology, Germany

Eberhard Karls Universität Tübingen, Germany 
Thierry Chanelière

Jean-Pierre Galaup

Ryszard Jankowiak

Fedor Jelezko

Jaak Kikas

Jürgen Köhler

H. Peter Lu

Sebastian Mackowski

Neil Manson

Alfred J. Meixner

William E. Moerner

Norio Murase

Andrei V. Naumov

Michel Orrit

Taras Plakhotnik

Aleksander Rebane

Vahid Sandoghdar

Ivan Scheblykin

Vinod Subramaniam

Toshiro Tani

Charles Thiel

Niek van Hulst

Martin Vacha

Silvia Völker
Paris-Sud University, France

Paris-Sud University, France

Kansas State University, Manhattan, USA

Ulm University, Germany

University of Tartu, Estonia

University of Bayreuth, Germany

Bowling Green State University, USA

Nicolaus Copernicus University, Toruń, Poland

Australian National University, Canberra, Australia

Eberhard Karls Universität Tübingen, Germany

Stanford University, USA

Natl Inst. of Adv. Industrial Sci. and Techn., Osaka, Japan

Institute for Spectroscopy RAS / MSPU, Moscow, Russia

Leiden University, Netherlands

Queensland University, Brisbane, Australia

Montana State University, Bozeman, USA

Max Planck Inst. for the Sci. of Light, Erlangen, Germany

Lund University, Sweden

University of Twente, Enschede, Netherlands

Tokyo Uni. of Agriculture and Technology, Tsukuba, Japan

Montana State University, Bozeman, USA

The Institute of Photonic Sciences, Barcelona, Spain

Tokyo Institute of Technology, Japan

Leiden University, Netherlands

\section{Local Organizing Committee}

HBSM-2018 was managed by the Interinstitutional scientific team working in laser selective spectroscopy and nanoscopy of single molecules, condensed matter and nanostructures (www.single-molecule.ru).

Andrei Naumov (head)

Kamil Karimullin (sci. secretary)

Victor Zadkov (ISAN section chairman)

Dmitry Isaev (MSPU section chairman)

Artem Arzhanov

Ivan Eremchev

Anna Es'kova

Svetlana Gladenkova

Maxim Gladush

Alina Golovanova

Alexey Gorshelev

Natalia Lozing

Konstantin Magaryan

Olga Morozova

Alexander Ruzaev

Alexander Savostianov
Institute for Spectroscopy RAS / MSPU / MIPT

Institute for Spectroscopy RAS / MSPU

Institute for Spectroscopy RAS / HSE / MSU

Moscow State Pedagogical University

Institute for Spectroscopy RAS / MSPU

Institute for Spectroscopy RAS / MIPT

Moscow State Pedagogical University

Moscow State Pedagogical University

Institute for Spectroscopy RAS / MSPU

Institute for Spectroscopy RAS / MSPU

Institute for Spectroscopy RAS

ISAN / Higher School of Economics

Moscow State Pedagogical University / ISAN

Moscow State Pedagogical University

Moscow State Pedagogical University

Institute for Spectroscopy RAS

Volunteers: Irina Alimkina (MSPU); Mikhail Gubin (VSU, MSPU); Maria Knyazeva (MSU); Elizaveta Kozhina (MSPU); Artur Nelubov (MIPT); Sergey Orlov (ISAN); Aleksei Prokhorov (VSU); Anna Pryakhina (MSPU); Eduard Podshivailov (MSU); Alyona Samsonova (MSPU); Ekaterina Smirnova (MIPT); Aleksandra Triznova (MSPU). 


\section{The Shpol'skii-Rebane-Personov Prize for outstanding scientific achievements in the area of site-selective spectroscopy}

The International Advisory and Program Committee of the International Conference on Hole Burning and Single Molecule Spectroscopy has established a special Prize and a medal named after Prof. Eduard Shpol'skii, Prof. Karl Rebane, and Prof. Roman Personov that will be awarded for outstanding scientific achievements in the area of site-selective spectroscopy. The Prize bears the names of three outstanding scientists - the founders of the scientific direction of selective spectroscopy of complex organic molecules.
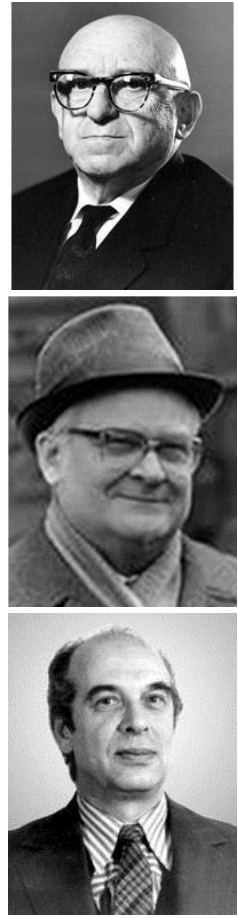

Prof. Eduard Shpol'skii and his colleagues from MPSU in 1952 discovered the effect of quasilinear spectra of organic chromophore molecules in the substances later named "Shpol'skii matrices" at cryogenic temperatures, which opened the way for the fine-structure spectral analysis of a huge number of complex organic compounds. The term "Shpol'skii effect" has now become a commonly used term in scientific communities around the world.

Prof. Karl Rebane, who headed the Institute of Physics of the Academy of Sciences of the Estonian SSR, and his colleagues made a great contribution to understanding the appearance the zero phonon spectral lines of single impurity molecules. He developed the theoretical foundations of optical spectroscopy of impurity centers, which subsequently led to the discovery of the narrow stable hole burning simultaneously in the Institute of Physics in Tartu and the Institute for Spectroscopy, USSR Academy of Sciences in 1974. Karl Rebane predicted and then discovered hot luminescence in crystals.

Prof. Roman Personov and his colleagues at the Institute for Spectroscopy of the USSR Academy of Sciences in 1972 were among the first to conduct unique experiments on laser narrowing of fluorescence lines in impurity organic compounds. Many authors both in Russia and worldwide call the effect of laser excitation of the finestructure luminescence spectra the "Personov effect".

The discoveries of these three scientists stimulated numerous studies in the area of site-selective spectroscopy all over the world, culminating in the detection of spectra of single molecules, which later played a decisive role in the emergence of a new interdisciplinary direction of ultra-high spatial resolution optical spectroscopy, now known as nanoscopy. Numerous investigations in this field have been the basis and the reasons for organizing the HBSM conference series.

Shpol'skii-Rebane-Personov Prize is awarded for the first time at HBSM-2018 to Prof. Fedor Jelezko (Ulm University, Germany) for his pioneering experiments on quantum optics with single molecules, on $N V$ color centers in diamonds and their applications for magnetometry and quantum information. Fedor Jelezko made full use of zero-phonon line of organic molecules and of color centers to test quantum optical concepts such as light shift, Rabi resonances, and to develop new applications for sensitive magnetometry. Prof. F. Jelezko has made groundbreaking contributions that have established atomic scale lattice defects (color centers) in diamond as a technology that can

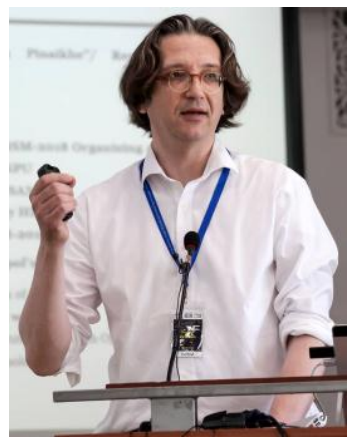
harness quantum coherence and entanglement even under ambient conditions. Furthermore, he has developed applications in such devices that are ranging from quantum information processing to the realization of new quantum sensing and imaging technologies. 


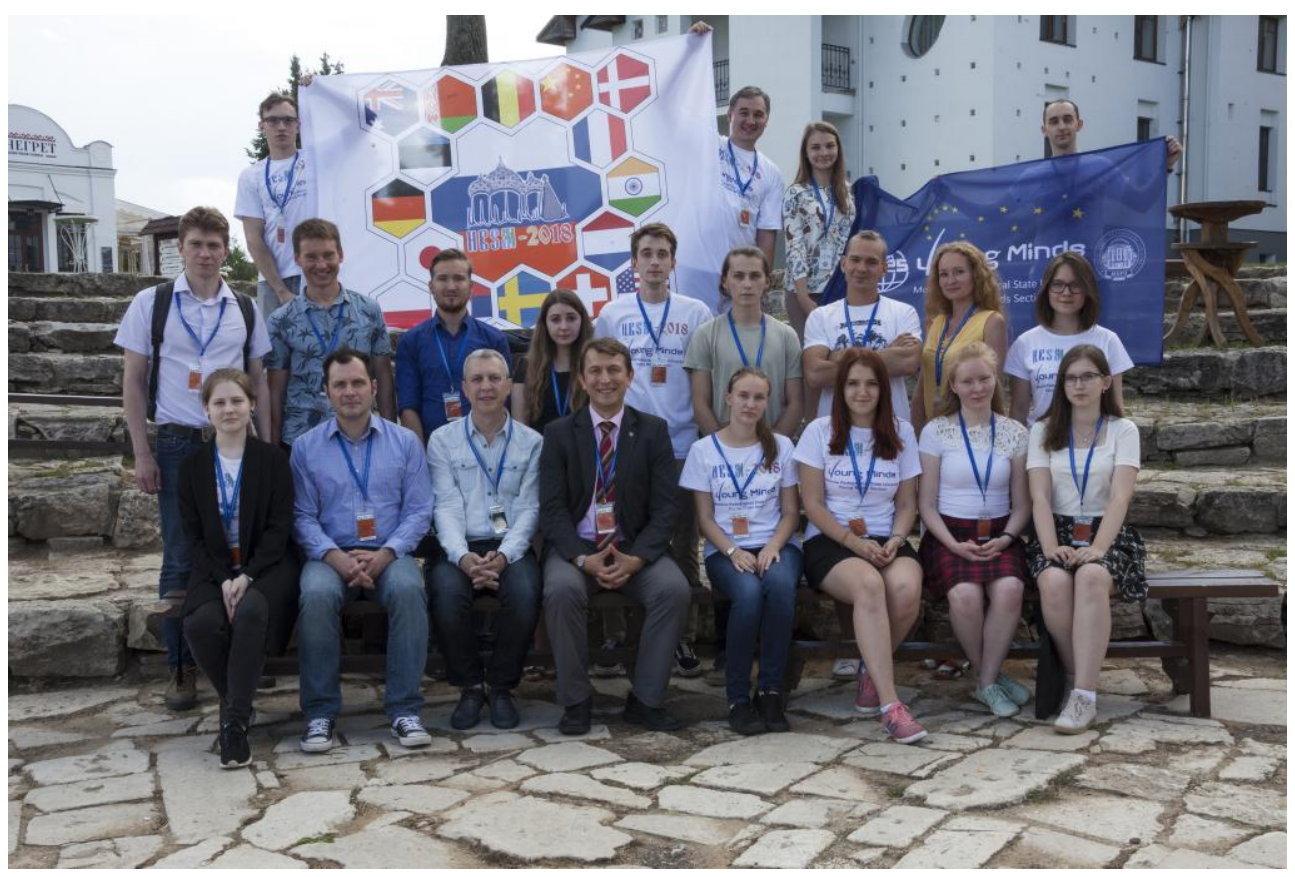

HBSM-2018 Local Organizing Committee

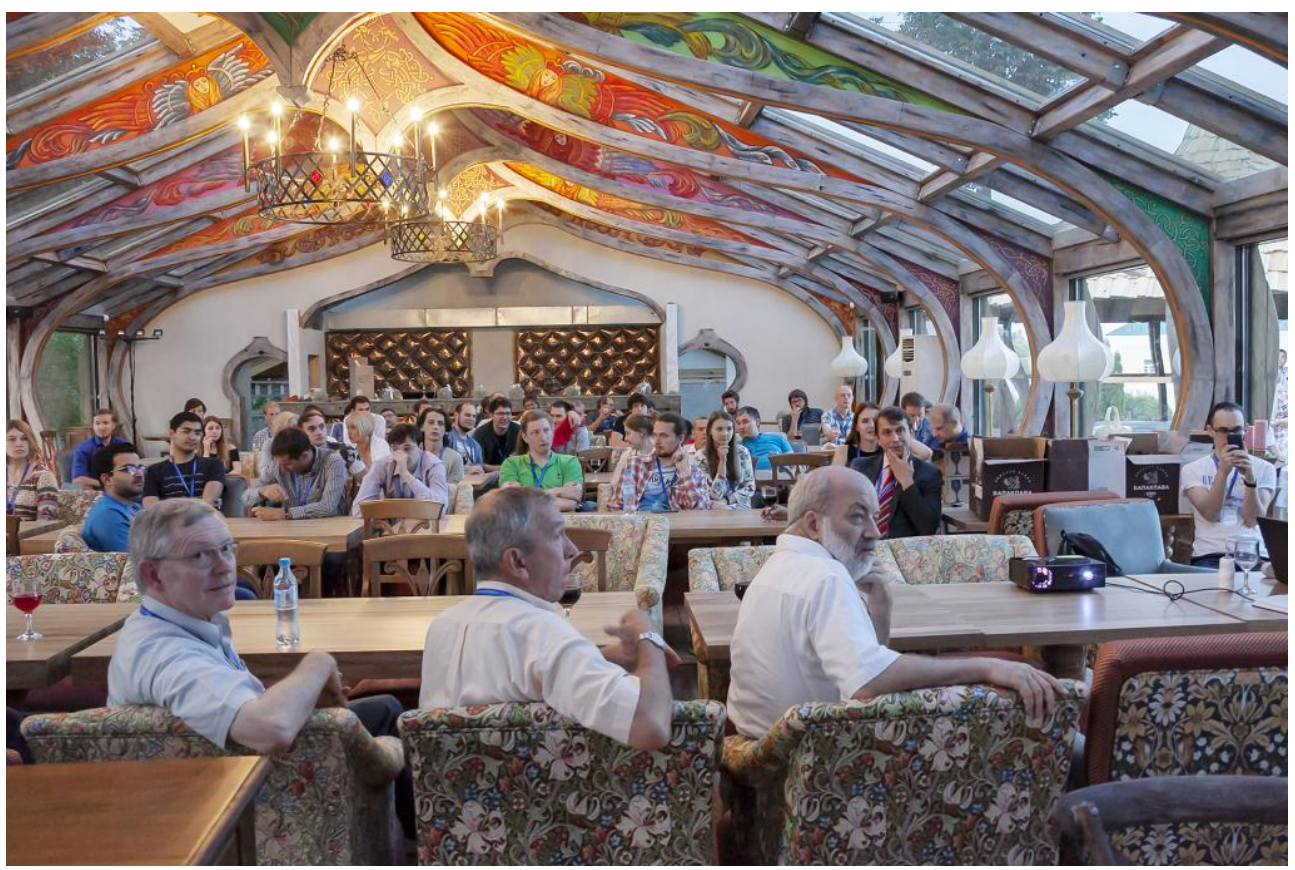

Photo from the EPS Young Minds meeting on HBSM-2018 


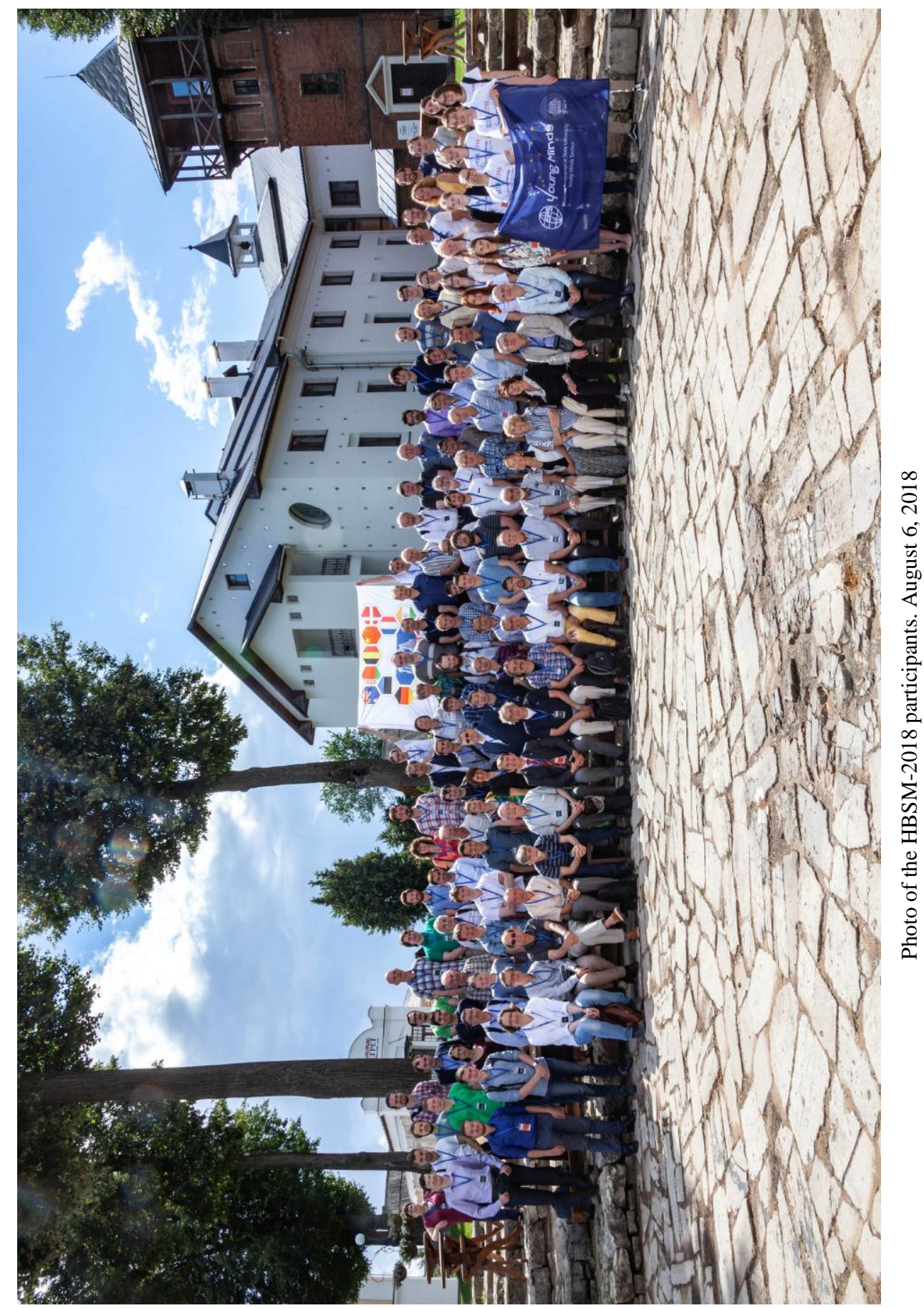

